\title{
Inclusión educativa en contextos de educación superior. Una revisión narrativa
}

\author{
Educational inclusion in the context of higher education. A \\ narrative review
}

\author{
Inclusão educacional em contextos da educação superior. \\ Uma revisão narrativa
}

\section{Irene Villalobos Saldivia*}

\begin{abstract}
RESUMEN
El presente artículo es una revisión narrativa, cuyo objetivo es identificar y analizar las investigaciones publicadas durante la última década con relación a la educación inclusiva en contextos de educación superior. Se emplea la técnica de análisis temático a los artículos seleccionados de Clarivate, Web of Science, Scopus, Ebsco, Scielo y Redalyc. Los resultados se agrupan en: 1 . Actores educativos, 2. Grupo disidente o grupo sujeto de vulneración de derechos y 3. Principales hallazgos. Las investigaciones se centran en estudiantes en discapacidad y en acciones de promoción para el acceso y permanencia en la educación. Los estudios son en su mayoría cualitativos, que dan cuenta de las experiencias y percepciones que tienen las y los estudiantes en su proceso formativo. Se concluye respecto de la necesidad de desarrollar un enfoque holístico que amplíe la mirada a toda la comunidad educativa en el abordaje de la educación inclusiva.
\end{abstract} Palabras clave:

\begin{abstract}
This article is a narrative review, which aims to identify and analyze research published over the last decade about inclusive education in the context of higher education. The technique of thematic analysis is used for articles selected from Clarivate, Web of Science, Scopus, Ebsco, Scielo and Redalyc. The results are
\end{abstract}

Keywords: inclusion, school integration programs, participation, 
grouped into: 1. educational actors, 2. dissident group or group subject to rights violations and 3. main findings. The research focuses on students with disabilities and on actions to promote access to and permanence in education. The studies are mostly qualitative, giving an account of the experiences and perceptions that students have in their educational process. It is concluded that there is a need to develop a holistic approach that broadens the view of the whole educational community in its approach to inclusive education.

\section{RESUMO}

Este artigo é uma revisão narrativa com o objetivo de identificar e analisar as pesquisas publicadas durante a última década em relação à educação inclusiva em contextos de ensino superior. É utilizada a técnica de análise temática dos artigos selecionados de Clarivate, Web of Science, Scopus, Ebsco, Scielo e Redalyc. Os resultados são agrupados em: 1. atores educativos; 2. grupo dissidente ou grupo sujeito de vulneração dos direitos e 3. principais achados. A pesquisa se concentra nos estudantes com deficiência e em ações para promover o acesso e a permanência na educação. Os estudos são, em sua maioria, qualitativos e relatam as experiências e percepções dos estudantes em seu processo formativo. A conclusão é que existe uma necessidade de desenvolver um enfoque holístico que amplie a visão de toda a comunidade educacional na abordagem da educação inclusiva. critical incidents, students.

Palavras-chave: Educação, inclusão educacional, educação superior, ensino superior, inclusão, diversidade. 


\section{Introducción}

La educación es un bien común de todas las personas, debido a que pretende y promueve el desarrollo del ser humano y otorga la posibilidad de contribuir a la sociedad a través del diálogo y el entendimiento entre diversas culturas. De esta manera, Blanco (2006), Bolívar (2012) y Ruiz (2020), estiman que el sentido fundamental de la educación es cultivar la humanidad, puesto que este derecho no solo remite a las personas a recibir educación sino, como todo derecho humano, también implica obligaciones estatales para ser garantizado. De acuerdo con Delors (1996), la educación es "uno de los principales medios disponibles para fomentar una forma más profunda y armoniosa del desarrollo humano y de ese modo, reducir la pobreza, la exclusión, la ignorancia y la guerra" (p. 11). A su vez, considera que "la educación tiene una doble misión: enseñar la diversidad de la especie humana y contribuir a una toma de conciencia de las semejanzas y la interdependencia entre todos los seres humanos" (p. 9).

Un punto de demarcación sociohistórico cultural es el reconocimiento de la educación como un derecho humano básico en la Declaración Universal de Derechos Humanos (1948). Este fue reafirmado en la Convención sobre los Derechos del Niño (1989), que ha sido ratificada casi universalmente (Ruiz, 2020). No obstante, en el mundo todavía existen personas para las que no se ha hecho efectivo este derecho, por lo que se ha configurado como un proceso paulatino en el tiempo.

Así, el derecho a la educación está inspirado por el principio de igualdad de oportunidades que, según la Convención sobre los Derechos del Niño, significa asegurar el acceso de todos los niños, sin ningún tipo de discriminación. Además, Ruiz (2020) explica que el acceso de la educación comprende el primer paso en el derecho a la educación, ya que busca promover el desarrollo y el aprendizaje a lo largo de toda la vida de las personas.

\section{Educación superior}

La Organización de las Naciones Unidas para la Educación, la Ciencia y la Cultura (UNESCO, 1998) sostiene que, desde fines del siglo XX, la educación superior se encuentra en proceso de construcción y reconstrucción por los constantes cambios sociopolíticos, económicos y laborales en 
todo el mundo. La educación terciaria experimenta procesos de transformación, que buscan responder a las necesidades sociales y promover la solidaridad, la igualdad y poner en el centro a las y los estudiantes para brindar la oportunidad, el acceso y la permanencia en la educación.

En el Pacto Internacional de Derechos Económicos, Sociales y Culturales de la Naciones Unidas, de 1956, artículo 13º, párrafo segundo, se establece que "la enseñanza superior debe hacerse igualmente accesible a todos, sobre la base de la capacidad de cada uno, por cuantos medios sean apropiados, y en particular por la implantación progresiva de la enseñanza gratuita" (Asamblea General de la Organización de las Naciones Unidas, 1966). De esta forma, las personas tienen acceso a la educación superior en función de sus méritos, lo que se encuentra en la Declaración Universal de Derechos Humanos, adoptada y proclamada por la Asamblea General de la Organización de las Naciones Unidas (ONU):

Toda persona tiene derecho a la educación. La educación debe ser gratuita, al menos en lo concerniente a la instrucción elemental y fundamental. La instrucción elemental será obligatoria. La instrucción técnica y profesional habrá de ser generalizada; el acceso a los estudios superiores será igual para todos, en función de los méritos respectivos. (1949, artículo 26)

En efecto, el acceso a la educación superior ha ido progresivamente en ascenso. Esto es fundamental para la promoción y el fortalecimiento del derecho a la educación como un derecho humano que está en la base de una sociedad más justa. Ruiz (2020) argumenta que "el principio de igualdad de reconocimiento y redistribución, así como de equidad en la conceptualización de las prestaciones educacionales pueden proveer indicadores válidos para la medición del derecho a la educación, en cada contexto socio-histórico particular" (p. 49). En consecuencia, la obligación de los Estados, en materia de derechos humanos, implica garantizar la disponibilidad, accesibilidad, aceptabilidad y adaptabilidad de la enseñanza (Tomasevski, 2002).

\section{Inclusión educativa en contexto de educación superior}

La UNESCO (2005) pone de manifiesto la idea de inclusión educativa, la cual debe sustentarse en los derechos humanos, en los que el acceso 
y participación a una educación de calidad es un imperativo. En este sentido, todos los seres humanos, independiente de su etnia, género, forma de aprender, entre otros, deberían gozar y ejercer el derecho a la educación. Por consiguiente, la educación inclusiva se entiende como una estrategia clave para alcanzar la educación universal (declaración que se aprobó desde 1990), partiendo del hecho de que la educación es un derecho humano básico y fundamental para constituir una sociedad más justa e igualitaria (UNESCO, 2009).

Desde esta perspectiva, la concepción de "inclusión educativa" se encuentra en directa relación con las oportunidades que ofrece cada país a sus ciudadanos. Cada Estado puede adoptar directrices que estimulen, promuevan y difundan el desarrollo de cada individuo, sin importar sus condiciones o características. Sin embargo, como expresa Díaz-Romero, (2006):

Entrar a la universidad es solo el comienzo de un extenso y difícil camino para los grupos postergados, ya que para culminar con éxito el pregrado deben sortear múltiples barreras, que prolongan sus carreras, aumentan sus tasas de deserción y los alejan de las nuevas prácticas y conocimientos en las disciplinas que estudian. (p. 33)

Es preciso recalcar que la inclusión ha sido referida no solo desde el criterio de acceso, sino también desde la accesibilidad, la cual no se agota en la mera presencia (acceso), sino también abarca la posibilidad de participación; por ejemplo, en el contexto de grupos en situación de discapacidad sensorial o motora. Por otra parte, Blanco (2006) considera que, para que sea posible el proceso de inclusión en este nivel, es fundamental "minimizar las múltiples barreras propias de la educación superior a fin de asegurar el acceso, la permanencia y el egreso" (p. 40).

La inclusión educativa, desde Arnaiz (2003), Ainscow (2005), Blanco (2006), Booth (2006) y Echeita (2006), se vincula con el acceso, la participación y los logros de todas y todos los estudiantes, poniendo especial énfasis en aquellos que se encuentran en riesgo de ser excluidos o marginados. Lo anterior implica adaptar la cultura, las políticas y las prácticas, para atender la diversidad de necesidades educativas y hacer efectivo el derecho de la no discriminación en las distintas esferas de la vida. De esa forma, todas las personas, sea cual sea su con- 
dición, puedan contar con mayores posibilidades para desarrollarse como tales y participar en la sociedad.

La inclusión en educación superior, desde la posición de Latorre, González y Espinoza (2009), ha movilizado a los organismos internacionales en la construcción de una serie de políticas de equidad orientadas a remediar los efectos de las desigualdades, y comprendido acciones referidas a permitir que todas las personas tengan acceso, permanencia, logros y resultados en el sistema educativo. En definitiva, estas estrategias buscan eliminar las barreras para el aprendizaje y promover la participación de todos los actores de la sociedad.

Desde el análisis de Torres (2011), ser incluido no es exclusivo de las escuelas, es también una forma concreta de participación y, específicamente, de cómo insertarse en la sociedad desde la perspectiva queer en pedagogía. Desde dicho enfoque, se invita a repensar las prácticas educativas y las relaciones de poder en el sistema educativo, a raíz de que estas promueven acciones formativas que reproducen y legitiman el discurso de lo hegemónico y excluyen todo lo demás. Además, Medina (2018) añade que las y los estudiantes no pueden considerarse incluidos en su totalidad hasta que no adquieran las habilidades y competencias necesarias y, para esto, es fundamental su inserción en la educación superior.

De igual modo, Blanco (1999) reflexiona sobre la inclusión como un proceso en el que se transforman las instituciones educativas y se entregan respuestas basadas en características y potencialidades de cada individuo. Asimismo, García (2013) concibe a la educación inclusiva como un conjunto de procesos, tanto individuales como colectivos, que se instalan al interior de las instituciones, cuyo fin es promover el desarrollo de todas las personas, en una cultura de respeto y reconocimiento de la dignidad intrínseca y de los derechos iguales e inalienables de todos los seres humanos.

En la educación inclusiva el objeto de innovación no recae en la persona estudiante, sino en el sistema educativo, en la institucionalidad y en la comunidad en general, porque se pone en el centro las barreras y cómo estas pueden generar menor o mayor riesgo de exclusión a las y los estudiantes. Por ende, este enfoque no se sitúa en las dificultades individuales, puesto que el foco está en conocer el tipo 
de oportunidades y apoyos que se brindan en el proceso formativo, a través de la práctica política y cultura institucional. Algunos ejemplos de barreras que propone Blanco (2006) y la UNESCO (2005) pueden ser la escasez de recursos materiales, la rigidez en la enseñanza, la formación de académicas y académicos, el escaso trabajo colaborativo, la falta de apoyo de la familia y los padres, entre otros.

García, García y Moreno (2012) concuerdan que todo acto educativo debe contemplar dos formas de responder al hecho de la diversidad: la respuesta positiva, que dice relación con el derecho de todo ser humano a ser diferente, y la negativa, que genera discriminación o desigualdad. La respuesta a la diversidad valiosa es la de diversidad en positivo, porque esta expresa y enriquece las posibilidades de todos los seres humanos.

En palabras de García, García y Moreno (2012), la “Diversidad es el conjunto, la mezcla colectiva de las diferencias y similitudes, la mezcla de minorías y mayorías; es decir [que] en la diversidad [entramos] todes (...)" (p. 20). Esta conceptualización reafirma la importancia de deconstruir el concepto de diversidad asociado a los grupos que son "diferentes" de lo normado y sus efectos excluyentes y violentos en otras categorías (González et al., 2019). Por lo tanto, Medina (2018) considera que la diversidad es un hecho que se da en los grupos sociales y que supone una oportunidad de aprender y mejorar para toda la comunidad educativa. Por este motivo, la UNESCO (2005) señala que:

El propósito de la educación inclusiva es permitir que los maestros y estudiantes se sientan cómodos ante la diversidad y la perciban no como un problema, sino como un desafío y una oportunidad para enriquecer las formas de enseñar y aprender. (p. 14)

La presente revisión se concentra en la inclusión educativa o educación para todos, considerando los postulados de Dyson (2001), que distingue cuatro variedades de inclusión: como ubicación, como educación para todos, como participación, y la inclusión social. Por eso, dentro de esta categoría se incorpora la diversidad de estudiantes que poseen derecho a ser educados y, por tanto, se clasifica en relación con esa diversidad. En primer lugar, la diversidad asociada a las personas en situación de discapacidad de tipo cognitiva o física y/o con necesidades educativas especiales (NEE). En segundo lugar, la diversidad 
vinculada a la inclusión social de estudiantes, referida a su contexto socioeconómico y/o vulnerabilidad. En tercer lugar, la diversidad sexual o de género. $Y$, por último, la diversidad étnica o racial.

Por todo lo expresado en párrafos anteriores, surge el interés de recopilar antecedentes sobre la inclusión educativa en el contexto de educación superior para elaborar una revisión que responda a la siguiente pregunta investigativa: ¿qué se ha estudiado sobre la inclusión educativa en contextos de educación superior? A partir de esta interrogante se propone el siguiente objetivo: identificar y analizar las investigaciones publicadas durante la última década con relación a la inclusión educativa en contextos de educación superior.

\section{Metodología}

Se realizó una revisión narrativa de tipo cualitativo que, según Rother (2007), tiene como características principales describir y discutir teórica y/o contextualmente el "estado del arte" de una temática en particular. Según la autora, este tipo de revisiones de la literatura cumplen un rol fundamental en la educación continua, porque permiten tanto adquirir como actualizar el conocimiento sobre un tema específico y de manera relativamente rápida.

La recopilación de información se realizó desde las siguientes plataformas digitales de revistas indexadas: Clarivate, Web of Science, Scopus, Ebsco, Scielo, y Redalyc. Las dos primeras recogen producción internacional de alto impacto y confiabilidad científica, mientras que las otras cuentan con amplitud de literatura de toda Latinoamérica.

Para llevar a cabo la búsqueda de los artículos, los criterios fueron los siguientes: 1) investigaciones empíricas que aborden inclusión educativa y educación superior; 2) artículos en América Latina, el Caribe y países iberoamericanos; y 3) cuyas publicaciones se encuentren entre los años 2010 a 2020.

Los descriptores utilizados para la búsqueda resultaron de las siguientes combinaciones de palabras claves: inclusión educativa AND educación superior, inclusión AND educación superior, inclusión AND enseñanza superior. Debido a que el descriptor "inclusión" por sí solo amplía los resultados donde se imparte educación primaria, secundaria y superior, se combinó mediante el operador booleano AND 
para asegurar la búsqueda de contenido relacionado con educación superior y sus derivados que son pertinentes a esta revisión.

En el primer caso se encontraron 92 artículos y en el segundo 22 artículos. En lo que respecta a la literatura científica encontrada, esta corresponde a criterios no empíricos relacionados con reflexiones teóricas, temas relativos a otros ámbitos de inclusión y/o análisis de políticas públicas. Por ello, fue necesario revisar cada artículo, hasta llegar a una selección de 11 estudios.

Para registrar los datos se organizó la información de cada artículo en los siguientes campos: autor (es), objetivo de estudio, grupo disidente/grupo sujeto de vulneración de derechos, actores educativos y principales resultados. A continuación se presenta, en la tabla 1, los estudios seleccionados que cumplen los criterios de búsqueda. Por lo tanto, estas categorías responden a los objetivos planteados para esta revisión y pretenden facilitar el análisis de los datos obtenidos. 


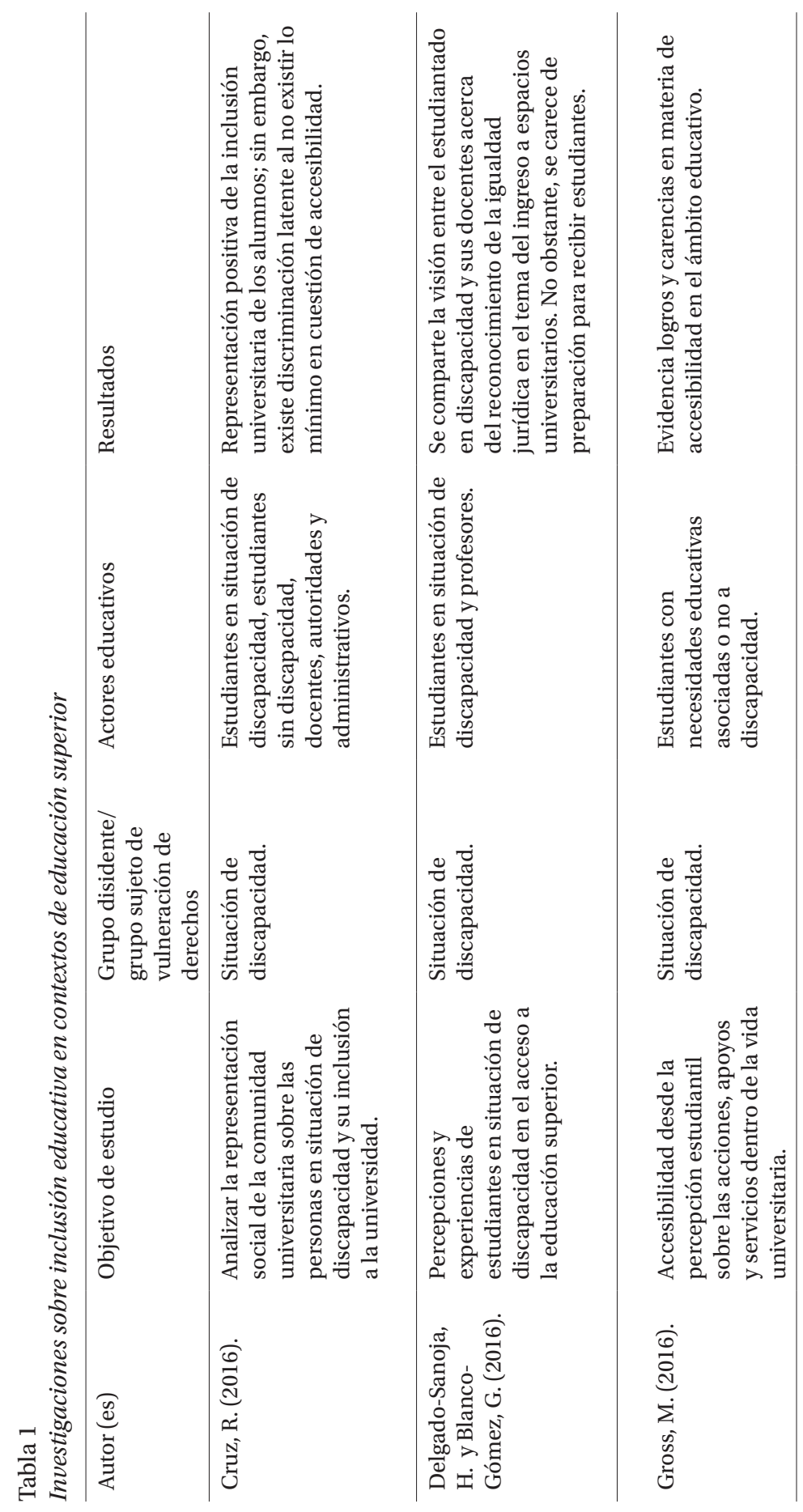



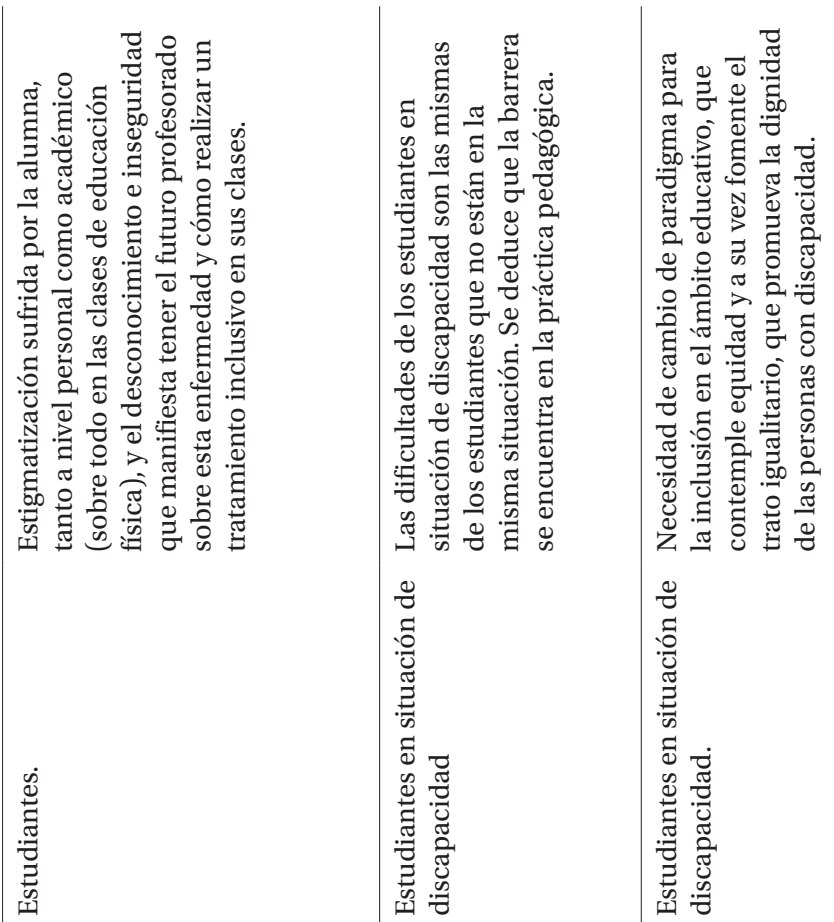

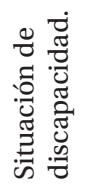

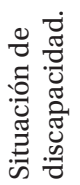

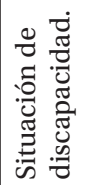

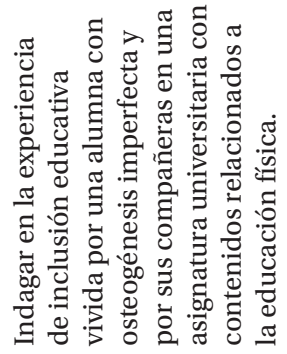

용

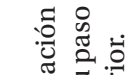

兽芯

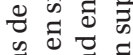

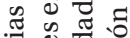

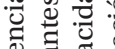

. ฮี ซ్ల

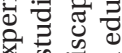

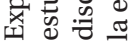
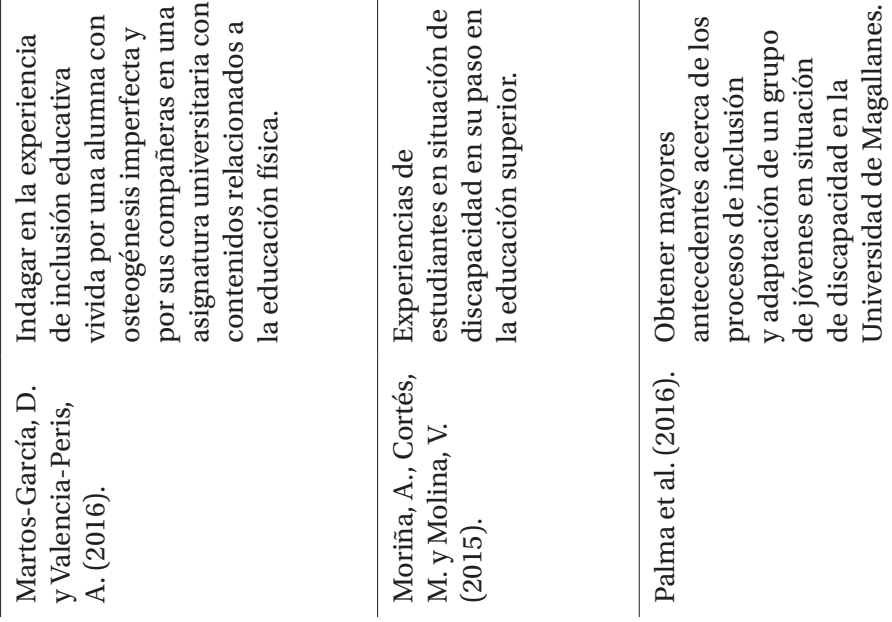


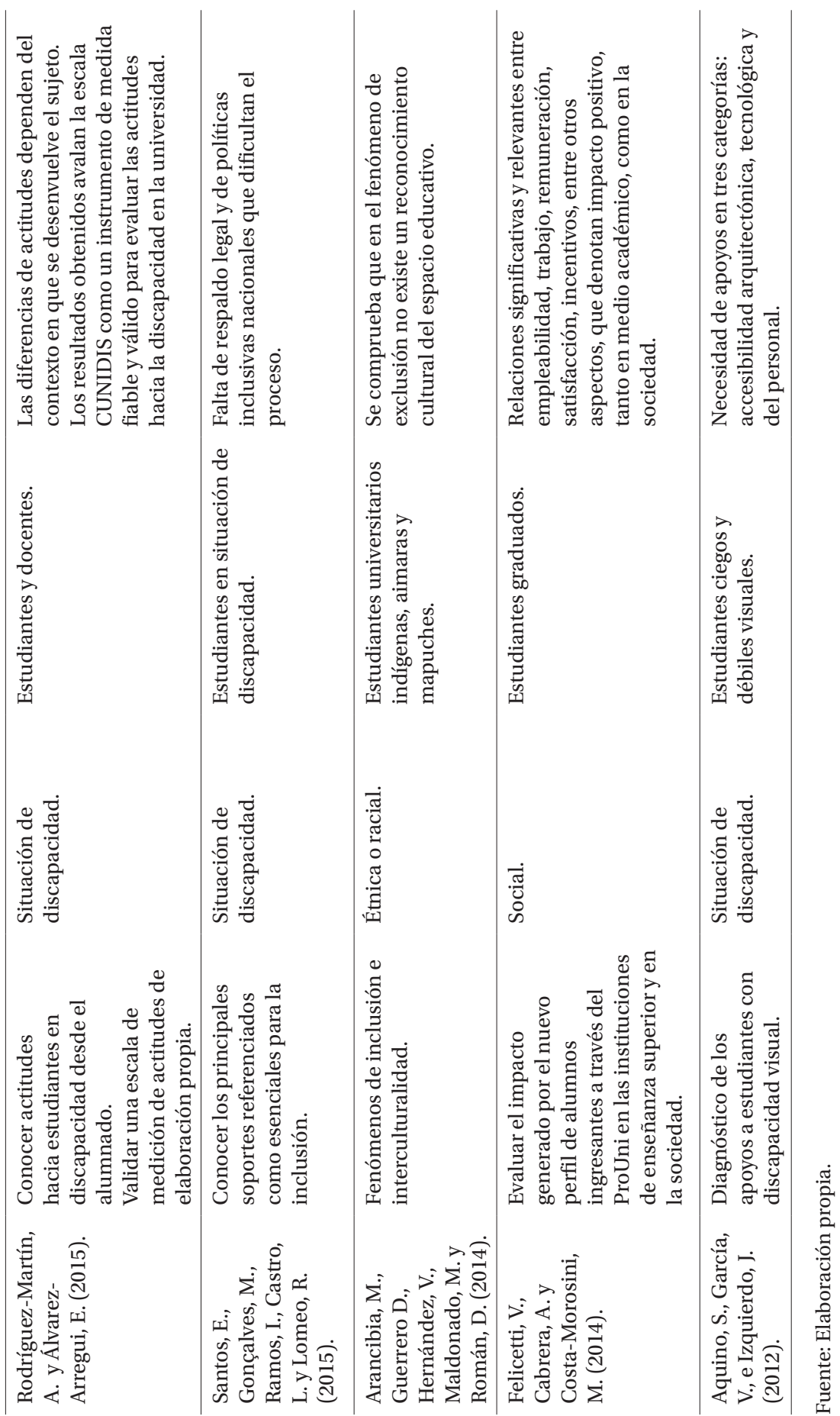




\section{Resultados}

De un corpus de 11 artículos, los países de origen son: Chile, Venezuela, España, Portugal, Costa Rica, México y Brasil. Los artículos seleccionados para la revisión corresponden a tres de Chile (Palma et al., 2016; Martos-García y Valencia-Peris, 2016; Arancibia et al., 2014); dos de México (Cruz, 2016; Aquino, García e Izquierdo, 2012); dos de España (Rodríguez-Martín y Álvarez-Arregui, 2015; Moriña, Cortés y Molina, 2015), un artículo de Costa Rica (Gross, 2016), uno de Venezuela (Delgado-Sanoja y Blanco-Gómez, 2016), uno de Portugal (Santos et al., 2015) y uno de Brasil (Felicetti, Cabrera y Costa-Morosini, 2014). Los artículos seleccionados se caracterizan por poseer fecha de publicación entre los años 2012 y 2016.

La principal metodología empleada por los artículos seleccionados es cualitativa, presente en ocho artículos (Delgado-Sanoja y BlancoGómez, 2016; Gross, 2016; Martos-García y Valencia-Peris, 2016; Arancibia et al., 2014; Santos et al., 2015; Moriña, Cortés y Molina, 2015; Palma et al., 2016; Aquino, García e Izquierdo, 2012). Por otro lado, hay un estudio de metodología mixta (Felicetti, Cabrera y Costa-Morosini, 2014) y dos de metodología cuantitativa (Cruz, 2016; Rodríguez-Martín y Álvarez-Arregui, 2015).

Es necesario señalar que, de los ocho estudios cualitativos, seis utilizaron la entrevista semiestructurada y/o en profundidad (Martos-García y Valencia-Peris, 2016; Palma et al., 2016; Delgado-Sanoja y Blanco-Gómez, 2016; Aquino, García e Izquierdo, 2012; Arancibia et al., 2014; Santos et al., 2015). Mientras que dos artículos emplearon otro tipo de técnica: Gross (2016) aplicó una encuesta en línea y organizó grupos focales para la recolección de datos; mientras que Moriña et al. (2015) usaron la técnica biográfico-narrativa con un análisis de datos estructural-narrativo. Otro hallazgo relevante consiste en que dos de los artículos responden a estudios de casos: Aquino et al. (2012) es un estudio de casos colectivos, en tanto Arancibia et al. (2014) se basa en un estudio de casos múltiples.

El artículo de Felicetti et al. (2014) usa metodología mixta. Por un lado, analiza frecuencias y relaciones sobre los datos recopilados y entrega una construcción a partir de las interfaces que surgieron del análisis de los textos descritos por los encuestados; por otro, profun- 
diza sobre la comprensión de los fenómenos investigados. En lo que respecta a los artículos que utilizaron una metodología cuantitativa se pueden mencionar dos: Cruz (2016) aplicó un cuestionario y Rodríguez-Martín y Álvarez-Arregui (2015) diseñaron un instrumento denominado Escala CUNIDIS (Cuestiones sobre Universidad y Discapacidad).

A continuación se realizó un análisis temático (Mieles, Tonon y Alvarado, 2012) de los títulos, resúmenes y palabras claves de estos textos, mediante el cual se logró identificar y analizar aspectos que han sido abordados en contextos de inclusión educativa por las instituciones de educación superior. A partir de esta revisión, se clasificaron de acuerdo con los temas que tratan, creando de manera inductiva tres ejes temáticos: 1) actores educativos, 2) grupo disidente o grupo sujeto de vulneración de derechos y 3) principales hallazgos.

\section{1) Actores educativos}

De un total de 11 artículos seleccionados sobre procesos de inclusión en la educación superior, se identificó que ocho estudios centran su mirada en las experiencias o perspectivas de estudiantes (Moriña et al., 2015; Arancibia et al., 2014; Gross, 2016; Aquino et al., 2012; Palma et al., 2016; Santos et al., 2015; Felicetti, Cabrera y Costa-Morosini, 2014; Martos-García y Valencia-Peris, 2016). En cambio, dos de los seleccionados (Rodríguez-Martín y Álvarez-Arregui, 2015; Delgado-Sanoja y Blanco-Gómez, 2016) desarrollaron un trabajo investigativo con estudiantes y docentes de la educación superior. Se debe destacar que solo el artículo de Cruz (2016) se interesó por diversos actores que conforman la comunidad educativa, entre los que se encuentran estudiantes, docentes, autoridades y equipo administrativo.

\section{2) Grupo disidente o grupo sujeto de vulneración de derechos}

La concepción de "inclusión" en contexto de educación superior es principalmente comprendida por nueve artículos con relación a temáticas de discapacidad o necesidades educativas especiales (MartosGarcía y Valencia-Peris, 2016; Cruz, 2016; Santos et al., 2015; Palma et al., 2016; Aquino et al., 2012; Gross, 2016; Rodríguez-Martín y ÁlvarezArregui, 2015; Moriña et al., 2015; Delgado-Sanoja y Blanco-Gómez, 2016). Dos artículos distan de lo anteriormente señalado; por un lado, 
se trabaja con la inclusión relativa a las etnias (Arancibia et al., 2014) y, por otro, se refiere a inclusión social (Felicetti, Cabrera y Costa-Morosini, 2014). Es necesario mencionar que, si bien en el apartado de introducción de la presente revisión se aludió a la inclusión relativa a diversidad sexual o de género, no se hallaron, no obstante, artículos que cumplieran con los criterios que se establecieron para este estudio.

\section{3) Principales hallazgos}

A partir del análisis de los 11 artículos seleccionados (Martos-García y Valencia-Peris, 2016; Cruz, 2016; Palma et al., 2016; Gross, 2016; Delgado-Sanoja y Blanco-Gómez, 2016, Rodríguez-Martín y Álvarez-Arregui, 2015; Moriña et al., 2015; Santos et al., 2015; Arancibia et al., 2014; Felicetti, Cabrera y Costa-Morosini, 2014; Aquino et al., 2012) se infiere que los países donde se contextualizan estas investigaciones, es decir, Chile, Venezuela, España, Portugal, Costa Rica, México y Brasil, cuentan con marcos normativos que promueven y protegen la libertad de educación en lo que refiere al acceso y a que este sea equitativo, lo que no necesariamente se hace coherente con la práctica y las experiencias que viven las y los estudiantes de educación superior.

Los artículos de Martos-García y Valencia-Peris (2016); Cruz (2016); Santos et al. (2015); Palma et al. (2016); Aquino et al. (2012); Gross (2016); Rodríguez-Martín y Álvarez-Arregui (2015); Moriña et al. (2015); Delgado-Sanoja y Blanco-Gómez (2016) se refieren a las dificultades que las y los estudiantes en situación de discapacidad se encuentran en su proceso de inserción a la educación superior, y a la posibilidad de continuar con sus estudios, los cuales aluden al criterio de accesibilidad más que al criterio de acceso de la educación superior. También se afirma en estos artículos que, a pesar de la ratificación de marcos regulatorios, las acciones y prácticas no han cambiado ni se han modificado a favor de las personas en situación de discapacidad, reconociéndose en los académicos una visión asistencialista motivada por la obligación legal.

Por otro lado, Moriña et al. (2015) reconocen que las dificultades de las y los estudiantes en situación de discapacidad son las mismas que las de estudiantes que no están en las mismas circunstancias. Se deduce que la barrera se encuentra en la práctica pedagógica de la educación superior, que no brinda los recursos para alcanzar la accesi- 
bilidad y superar dicha dificultad. Para esto, dichos autores proponen que el profesorado muestre una actitud positiva hacia la discapacidad, fomente prácticas inclusivas mediante metodologías alternativas a la lección magistral, realice adaptaciones curriculares, incorpore el uso de tecnologías en las aulas y se forme en temas relacionados con la adecuada atención a las necesidades derivadas de la discapacidad.

Por su parte, el estudio de Martos-García y Valencia-Peris (2016) afirma que la mayoría del estudiantado universitario en discapacidad no logra insertarse lo suficiente en grupos de pares y pocos de ellos participan en actividades extraprogramáticas, porque invierten la mayoría del tiempo en sus estudios con la finalidad de nivelarse y poder rendir académicamente. Por eso, dejan muy poco o nulo espacio para actividades de esparcimiento que les permitan o favorezcan formar vínculos profundos, como de amistad o relaciones de pareja dentro del ambiente universitario.

En cambio, el trabajo de Arancibia et al. (2014) se centra en las experiencias de cuatro estudiantes universitarios indígenas en Chile, y sus principales hallazgos radican en que no se genera un reconocimiento sociohistórico cultural en el espacio educativo, ni tampoco se integran prácticas multiculturales en el proceso formativo. Por lo tanto, este estudio concluye que, dentro de la institucionalidad, se ejercen acciones de exclusión y marginación con las personas que pertenecen a los pueblos indígenas. Por esta razón, se enfatiza la necesidad de legitimar el reconocimiento de las diversas etnias, poniéndolas al mismo nivel de las otras culturas presentes en la sociedad. Asimismo, este artículo promueve formular nuevas estrategias y dispositivos en la vinculación del Estado con la institución de educación superior y los pueblos originarios.

Sin embargo, desde la perspectiva de profesores y estudiantes, la investigación de Rodríguez-Martín y Álvarez-Arregui (2015) reporta que los actores estudiados poseen una actitud positiva ante la inclusión, destacando el equipo docente en comparación con los estudiantes. Además, se informa que las diferencias de actitud se producen, específicamente, por el colectivo y la rama de conocimiento a la que pertenecen. Así, una posible explicación es que las actitudes se encuentran condicionadas por la realidad en la que interactúan, y según la naturaleza de las propias titulaciones que cursan las y los estudiantes o donde 
imparte docencia el cuerpo académico. En otras palabras, según estos autores las diferencias que se pueden generar entre los actores involucrados no son en ningún caso individuales, sino que responden al perfil del área de estudio o de trabajo.

Por último, los artículos de Gross (2016) y Aquino et al. (2012) desarrollan un análisis y reflexión sobre la relevancia de promover, fortalecer y difundir la accesibilidad en contextos de educación superior. Por un lado, Gross (2016) advierte que la percepción de logros y carencias se vincula con materia de accesibilidad, la cual se relaciona directamente con el derecho a la educación, puesto que se aprecia un escaso compromiso institucional por parte del Estado. Por otro lado, Aquino et al. (2012) proponen robustecer tres áreas para fortalecer la accesibilidad de la educación superior: accesibilidad arquitectónica, tecnológica y del personal, las cuales deben ser promovidas por la universidad en concordancia con la experiencia de las y los estudiantes.

\section{Conclusión}

La presente revisión narrativa informa acerca de estudios de inclusión educativa en contextos de educación superior, realizados entre 2012 y 2016 en Chile, Venezuela, España, Portugal, Costa Rica, México y Brasil. Por lo tanto, recopila información y aprendizajes que pueden ser relevantes para generar políticas públicas, tanto a nivel local como internacional, y promover discusiones sobre intervenciones, programas, proyectos, protocolos y lineamientos de trabajo para el perfeccionamiento de estrategias educativas sobre inclusión en las instituciones de educación superior.

Es interesante señalar que la mayoría de los artículos centran su mirada en las y los estudiantes como actores claves, los cuales son capaces de reflexionar y opinar respecto a sus experiencias vividas en la educación superior. De esta forma, el resto de los actores de la comunidad educativa, como los docentes y/o académicos, han quedado relegados a un segundo plano, principalmente sobre las experiencias que vive el estudiantado y cómo se han ido aplicando las políticas y leyes de inclusión vigentes en gran parte de los países donde se sitúan estos artículos. Así, se entrega un análisis acerca de cómo es el proceso de prosecución de estudios de los estudiantes, y cómo estos son mirados y recibidos por el resto de la comunidad educativa. 
La UNESCO (2005), Blanco (1999), García (2013) y Medina (2018) estiman que el objetivo de la educación inclusiva es promover a toda la comunidad educativa a sentirse cómoda ante la diversidad y que esta no sea vista como un problema, sino como una oportunidad para enriquecer las formas de enseñar y aprender. Esto se presenta como un hallazgo esperado por parte de las investigaciones de Martos-García y Valencia-Peris (2016); Cruz (2016); Palma et al. (2016); Gross (2016); Delgado-Sanoja y Blanco-Gómez (2016), Rodríguez-Martín y ÁlvarezArregui (2015); Moriña et al. (2015); Santos et al. (2015); Arancibia et al. (2014); Felicetti, Cabrera y Costa-Morosini (2014); y Aquino et al. (2012), debido a que estos autores manifiestan que las barreras para la inclusión pueden disminuir significativamente si se realiza un trabajo colaborativo con todos los miembros que conforman la comunidad educativa de la enseñanza superior.

Por otro lado, los artículos de Rodríguez-Martín y Álvarez-Arregui (2015) y Delgado-Sanoja y Blanco-Gómez (2016) destacan el rol de las y los docentes en el proceso formativo de estudiantes en situación de discapacidad, puesto que son considerados como agentes claves para acompañar su inserción en los cursos, brindar un proceso flexible de enseñanza-aprendizaje e innovar en sus estrategias metodológicas, lo cual permitiría disminuir las barreras de acceso y permanencia en la educación superior. Por esta razón, realizar investigaciones con el equipo docente es imprescindible en los procesos educativos, por lo que se vuelve indispensable rescatar sus reflexiones, experiencias y opiniones respecto a los procesos de inclusión, dado que, en muchos casos, depende de ellos cómo se pueda llevar a cabo en el contexto de aula. Por lo tanto, esta temática se presenta como un desafío investigativo, dada la escasez de estudios que incorporan a las y los docentes universitarios como foco de estudio.

En la medida en que se considere a todos los actores involucrados en el contexto educativo, como lo expresan Ainscow (2005), Arnaiz (2003), Blanco (2006), Booth (2006) y Echeita (2006), se podrá llegar a mejores resultados para atender la diversidad educativa y velar por el derecho a la no discriminación en las distintas esferas de desarrollo del ser humano. Cabe destacar que, dentro de los artículos seleccionados, el estudio de Cruz (2016) es el único que se interesó por diversos actores que conforman la comunidad educativa: estudiantes, 
docentes, autoridades y equipo administrativo, lo que permite ampliar la mirada sobre la inclusión educativa en contexto de educación superior.

Por este motivo, estudiar el proceso de la inclusión educativa, como proponen Delgado-Sanoja y Blanco-Gómez (2016), implica escuchar a todos los actores desde sus expectativas, sus experiencias sobre la calidad de servicios que reciben, sus demandas y necesidades. Todo lo anterior es necesario conjugarlo con los informes oficiales emanados de las instituciones, contrastarlos con los datos estadísticos recogidos local, regional, nacional e internacional, y con el pronunciamiento de las instituciones que, en su calidad de ejecutores, velen por las prácticas inclusivas.

Latorre, González y Espinoza (2009) concuerdan que una forma de abordar la inclusión en educación superior es a través de políticas que promuevan la equidad, con el objeto de remediar las desigualdades y operacionalizar acciones para que todas las personas tengan acceso, permanencia, logros y resultados en el sistema educativo. Tal como expone Ottone (2006), "aunque la solución a los problemas de la pobreza e inclusión social es una tarea indelegable del Estado, la participación ciudadana y de la sociedad civil permiten enriquecer la iniciativa estatal" (en Díaz-Romero, 2006, p. 22). Por tanto, Blanco (2006) concluye que la inclusión en educación es una cuestión de justicia y equidad, que aspira a una educación de calidad para todas y todos, ya que responde a toda la sociedad.

La información obtenida a través de esta revisión narrativa contribuye a nuevas interrogantes para futuras investigaciones respecto de las expectativas que surgen en torno al rol que desempeña la institución de educación superior en la inclusión educativa y la participación de toda la comunidad, ello debido a que las instituciones de educación superior podrían ser una brújula para la comprensión de los procesos de inclusión educativa desde todos los participantes que la conforman. En palabras de García, García y Moreno (2012), en la diversidad entramos "todes". Por eso, la relevancia de deconstruir los conceptos de "inclusión" y "diversidad" asociados a grupos que se considera "distintos o diferentes" de lo normado, puesto que traen aparejado consecuencias excluyentes y violentas en otras dimensiones. Por último, la limitación de este estudio fue que la gran parte de los artículos selec- 
cionados se concentraron exclusivamente en la inclusión de personas en situación de discapacidad, siendo escasos los estudios sobre diversidad de etnia y diversidad social, dejando de lado otras diversidades, como las sexuales y de género.

Por último, esta revisión presenta hallazgos que matizan la concepción de "inclusión educativa" desde un contexto de educación superior, que permiten promover futuras investigaciones que aborden temáticas explícitas en apoyar los intereses de personas y comunidades marginadas o excluidas. Tal como postula Paradis (2000), la investigación es una forma de ejercer resistencia por medio del conocimiento, por lo que es necesario estudiar fenómenos psicológicos que contribuyan a la comprensión desde las distintas dimensiones de inclusión, diversidad y equidad en la educación.

\section{Referencias}

Ainscow, M. (2005) Developing inclusive education systems: what are the levers for change? Journal of Educational Change, 6(2), 109-124. DOI: https://doi.org/10.1007/s10833-005-1298-4

Aquino, S., García, V. e Izquierdo, J. (2012). La inclusión educativa de ciegos y baja visión en el nivel superior: Un estudio de caso. Sinéctica, (39), 01-21. Recuperado de https://www.redalyc.org/articulo.oa?id=99826889007

Arancibia, M., Guerrero D., Hernández, V., Maldonado, M. y Román, D. (2014). Análisis de los significados de estudiantes universitarios indígenas en torno a su proceso de inclusión a la educación superior. Psicoperspectivas, 13(1), 35-45. DOI: 10.5027/ psicoperspectivas-Vol13-Issuel-fulltext-255

Arnaiz, P. (2003). Educación inclusiva: una escuela para todos. Ediciones Aljibe.

Asamblea General de la Organización de las Naciones Unidas. (1948). Declaración Universal de los Derechos Humanos (217 [III] A).

Asamblea General de la Organización de las Naciones Unidas. (1966). Pacto Internacional de Derechos Civiles y Políticos (2200 [XXI] A).

Blanco, R. (1999). Hacia una inclusión para todos y con todos. En R. Blanco, Hacia una inclusión para todos y con todos (p. 3). Santiago, Chile: OREALC/UNESCO. 
Blanco, R. (2006) Inclusión en la Educación Superior. En P. Díaz-Romero (Ed.), Caminos para la Inclusión en la Educación Superior en Chile. Fundación Equitas.

Blanco, R. (2006). La inclusión en educación: una cuestión de justicia y de igualdad. Revista Electrónica Sinéctica, (29), 19-27. Recuperado de https://www.redalyc.org/articulo. oa?id=99815739003

Blanco, R. (2006). La equidad y la inclusión social: uno de los desafíos de la educación y la escuela hoy. Revista Iberoamericana sobre Calidad, Eficacia y Cambio en Educación, 4(3), 1-15. Recuperado de https://www.redalyc.org/articulo. oa?id=55140302

Bolívar, A. (2012). Justicia social y equidad escolar. Una revisión actual. Revista Internacional de Educación para la Justicia Social (RIEJS). 1(1). 9-45. Recuperado de https://revistas.uam.es/ riejs/article/view/308

Booth, T. (2006). Manteniendo el futuro con vida; convirtiendo los valores de la inclusión en acciones. En M. Verdugo (Ed.), Rompiendo inercias: claves para avanzar (pp. 211-217). Amarú.

Cruz, R. (2016). Discapacidad y educación superior: ¿Una cuestión de derechos o buenas voluntades? Revista de Investigación Educativa, (23), 1-23. Recuperado de http://www.scielo.org.mx/ scielo.php?pid=S1870-53082016000200002\&script=sci_abstract

Delgado-Sanoja, H. y Blanco-Gómez, G. (2016). Inclusión en la educación universitaria. Las palabras y experiencias detrás del proceso. Revista Electrónica Educare, 20(2), 163-180. DOI: http://dx.doi.org/10.15359/ree.20-2.9

Delors, J. (1996). La educación encierra un tesoro. Santiago, Chile: Ediciones Santillana.

Díaz-Romero, P. (2006). Caminos para la inclusión en la educación superior. Fundación Equitas.

Díaz-Romero, P. (2006). Lograr sociedades más equitativas implica dar mayores oportunidades educativas a los grupos más postergados. En P. Díaz Romero (Ed.), Caminos para la Inclusión en la Educación Superior en Chile. Fundación Equitas.

Dyson, A. (2001). Dilemas, contradicciones y variedades en la inclusión. En M. Verdugo (Ed.), Apoyos, autodeterminación y calidad de vida: actas de las IV Jornadas de Investigación sobre 
personas con discapacidad [Salamanca, 15-17 de mayo de 2001] (pp. 145-160). Amarú.

Echeita, G. (2006). Educación para la inclusión o educación sin exclusiones. Narcea.

Felicetti, V., Cabrera, F. y Costa-Morosini, M. (2014). Aluno ProUni: impacto na instituição de educação superior e na sociedade. Revista iberoamericana de educación superior, 5(13), 2139. DOI: https://doi.org/10.1016/S2007-2872(14)71951-1

García, G. (2013). Red nacional de educación superior inclusiva en Chile: contexto y misión. En L. Pérez, A. Fernández y S. Katz, (Comps.), Discapacidad en Latinoamérica: Voces y experiencias universitarias (pp. 133-148). Editorial de la Universidad de La Plata.

García, R., García, J. y Moreno, I. (2012). Estrategias de atención a la diversidad cultural en educación. Catarata.

González, A., Moreno, Y., Dinis, N., Sánchez, M., Penna, M. y Platero, L. (2019). Pedagogías Queer. Centro de Estudios Latinoamericanos de Educación Inclusiva. Recuperado de https://www. aacademica.org/aldo.ocampo.gonzalez/32

Gross, M. (2016). Accesibilidad al proceso educativo en el entorno universitario. Revista Electrónica: Actualidades Investigativas en Educación, 16(1), 1-17. DOI: http://dx.doi.org/10.15517/aie. v16i1.21920

Latorre, C., González, L. y Espinoza, O. (2009). Equidad en educación superior: Evaluación de las políticas públicas de la Concertación. Editorial Catalonia/Fundación Equitas.

Martos-García, D. y Valencia-Peris, A. (2016). Osteogénesis imperfecta y educación física. Un caso inédito de inclusión educativa. Estudios Pedagógicos, 42(1), 159-175. DOI: http://dx.doi. org/10.4067/S0718-07052016000100010

Medina, R. (2018). Inclusión y convivencia para prevenir el racismo, la xenofobia y otras formas de intolerancia: uno de los retos principales de nuestro sistema educativo. Ciencia, Técnica y Mainstreaming Social, (2), 53-65. DOI: https://doi. org/10.4995/citecma.2018.9852

Mieles, M., Tonon, G. y Alvarado, S. (2012). Investigación cualitativa: el análisis temático para el tratamiento de la información desde el enfoque de la fenomenología social. Universitas Humanís- 
ticas, (74), 195-225. Recuperado de http://revistas.javeriana. edu.co/index.php/univhumanistica/article/view/3648

Moriña, A., Cortés, M. y Molina, V. (2015). Educación inclusiva en la enseñanza superior: soñando al profesorado ideal. Revista Latinoamericana, 9(2), 161-175.

Organización de las Naciones Unidas para la Educación, la Ciencia y la Cultura. (1994). Informe Final de la Conferencia Mundial: Necesidades Educativas Especiales. Acceso y Calidad. UNESCO/MEC.

Organización de las Naciones Unidas para la Educación, la Ciencia y la Cultura. (1994). Necesidades especiales en el aula. París: UNESCO.

Organización de las Naciones Unidas para la Educación, la Ciencia y la Cultura. (1998). Declaración mundial sobre la educación superior en el siglo XXI: visión y acción. Educación superior y sociedad, 9(2), 97-113. Recuperado de https://www.iesalc. unesco.org/ess/index.php/ess3/article/view/171/162

Organización de las Naciones Unidas para la Educación, la Ciencia y la Cultura. (2005). Guidelines for inclusion: Ensuring Access to Education for All. UNESCO.

Organización de las Naciones Unidas para la Educación, la Ciencia y la Cultura. (2005). Orientaciones para la Inclusión: Asegurar el Acceso a la Educación para Todos. UNESCO. Recuperado de https://unesco.org/educacion/inclusive

Organización de las Naciones Unidas para la Educación, la Ciencia y la Cultura. (2008). La Educación Inclusiva: El Camino hacia el Futuro. En Conferencia Internacional de Educación (pp. 1 - 37). UNESCO.

Organización de las Naciones Unidas para la Educación, la Ciencia y la Cultura. (2009). Directrices sobre políticas de inclusión en la educación. Recuperado de http://unesdoc.unesco.org/ images/0017/001778/177849s.pdf

Palma, O., Soto, X., Barría, C., Lucero, X., Mella, D., Santana, Y. y Seguel, E. (2016). Estudio cualitativo del proceso de adaptación e inclusión de un grupo de estudiantes de educación superior con discapacidad de la Universidad de Magallanes. Magallania, 44(2), 131-158. DOI: http://dx.doi.org/10.4067/S071822442016000200007 
Paradis, E. (2000). Feminist and community psychology ethics in research with homeless women. American Journal of Community Psychology, 28(6), 839-858. DOI: https://doi. org/10.1023/A:1005115901078

Rodríguez-Martin, A. y Álvarez-Arregui, E. (2015). Universidad y discapacidad. Actitudes del profesorado y de estudiantes. Perfiles educativos, 37(147), 86-102. Recuperado de http:// www.scielo.org.mx/scielo.php?script=sci_arttext\&pid $=$ S0185-26982015000100006

Rother, E. (2007). Revisión sistemática x revisión narrativa. Acta Paulista de Enfermagem, 20(2). Recuperado de http://www.scielo. br/pdf/ape/v20n2/es_a01v20n2.pdf

Ruiz, G. (2020). El derecho a la educación: Definiciones, normativas y políticas públicas revisadas. EUDEBA.

Santos, E., Gonçalves, M., Ramos, I., Castro, L. y Lomeo, R. (2015). Inclusão no Ensino Superior: Perceções dos estudantes com Necessidades Educativas Especiais sobre o ingresso à universidade. Revista Portuguesa De Educação, 28(2), 251-270. DOI: https://doi.org/10.21814/rpe.7741

Tomasevski, K. (2002). Contenido y vigencia del derecho a la educación. Revista IIDH, (36), 15-38. Recuperado de https://revistas-colaboracion.juridicas.unam.mx/index.php/rev-instituto-interamericano-dh/article/view/34117/31076

Torres, G. (2011). Acerca de los usos, dificultades y posibilidades de la pedagogía queer. En Actas del IV Coloquio Internacional Interdisciplinario. Universidad de Buenos Aires. 